\title{
RELACIÓN ENTRE LAS PERCEPCIONES DE JUSTICIA ORGANIZACIONAL Y EL NIVEL DE COMPROMISO ORGANIZACIONAL EN LOS DOCENTES DE LA PUCE MATRIZ QUITO
}

RELATIONSHIP BETWEEN THE PERCEPTIONS OF ORGANIZATIONAL JUSTICE AND THE LEVEL OF ORGANIZATIONAL COMMITMENT IN THE TEACHERS OF THE PUCE MATRIZ QUITO

\author{
KATIA NARANJO ${ }^{1}$ \\ CARLA HIDROVO ${ }^{2}$
}

Recibido: 15 de mayo de 2017

Aceptado: 29 de agosto de 2017

\footnotetext{
${ }^{1}$ Pontificia Universidad Católica del Ecuador, Facultad de Psicología, Quito, Ecuador (kvnaranjo@puce.edu.ec).

${ }^{2}$ Pontificia Universidad Católica del Ecuador, Facultad de Psicología, Quito, Ecuador (carlita8808@hotmail.com).
} 



\title{
RELACIÓN ENTRE LAS PERCEPCIONES DE JUSTICIA ORGANIZACIONAL Y EL NIVEL DE COMPROMISO ORGANIZACIONAL EN LOS DOCENTES DE LA PUCE MATRIZ QUITO
}

\author{
RELATIONSHIP BETWEEN THE PERCEPTIONS \\ OF ORGANIZATIONAL JUSTICE AND THE LEVEL \\ OF ORGANIZATIONAL COMMITMENT IN THE \\ TEACHERS OF THE PUCE MATRIZ QUITO
}

Katia Naranjo y Carla Hidrovo

Palabras clave: justicia organizacional, compromiso organizacional, correlación, percepción.

Key words: organizational justice, organizational compromise correlation, perception.

\section{RESUMEN}

Este trabajo analizará si existe o no, una correlación directa entre las variables de justicia organizacional y compromiso organizacional. Para identificar la frecuencia con la que se presentan las variables, tanto de justicia como de compromiso organizacional, se aplicaron dos cuestionarios en una muestra de 278 docentes de varias facultades de la Pontificia Universidad Católica del Ecuador, 
matriz Quito. De esta manera, se realizó un diagnóstico identificando el tipo de variable que predomina entre los docentes de la institución.

El capítulo I aborda la necesidad de generar una investigación que analice si existe una correlación directa entre justicia y compromiso organizacional en los docentes de PUCE y que los resultados obtenidos sean considerados para la toma de decisiones en la institución.

El capítulo II comprende el marco teórico y marco conceptual, base para la ejecución y desarrollo de la investigación.
En el capítulo III, en el marco metodológico se presenta la definición y caracterización de la muestra, la definición del instrumento por aplicar y la validación del mismo.

En el capítulo IV se analiza e interpreta los resultados mediante una serie de técnicas como el análisis de Cronbach, la frecuencia, análisis de datos de contraste de hipótesis, correlación, regresión y análisis de datos demográficos.

En el capítulo $V$ se presentan las conclusiones y recomendaciones sobre la correlación entre las dos variables analizadas.

\section{ABSTRACT}

This paper will analyze whether or not there is a direct correlation between the variables of organizational justice and organizational commitment. In order to identify the frequency with which the variables of both justice and organizational commitment are presented, two questionnaires were applied in a sample of 278 teachers from various faculties of the Pontificia Universidad Católica del Ecuador, head office Quito. In this way a diagnosis was made identifying the type of variable that predominates among the institution's teachers.

Chapter I addresses the need to generate research that analyzes wheth- er there is a direct correlation between justice and organizational commitment in PUCE teachers and that the results obtained are considered for decision-making in the institution.

Chapter II includes the theoretical framework and conceptual framework, the basis for the execution and development of research.

In Chapter III, the methodological framework presents the definition and characterization of the sample, the definition of the instrument to be applied and the validation of the same.

In Chapter IV we analyze and interpret the results using a series of 
techniques such as Cronbach's analysis, frequency, hypothesis contrast data analysis, correlation, regression and demographic data analysis.
Chapter $V$ presents the conclusions and recommendations on the correlation between the two variables analyzed.

\section{INTRODUCCIÓN}

El presente artículo pretende establecer un hilo conductor para posteriores investigaciones relacionadas al análisis organizacional e institucional, ya que a partir de esta se ha generado un aporte para identificar cómo las percepciones de justicia en una muestra de 278 docentes infieren de manera directa con el compromiso organizacional, y partiendo de esto se puedan generar propuestas que permitan incrementar el nivel de compromiso en los docentes estableciendo mecanismos que ayuden a mejorar la percepción de justicia, así también se podría determinar las percepciones de justicia y el nivel de relación con el compromiso en las diferentes sedes de la universidad y en el personal administrativo, y con esto trabajar en mecanismos de acción que fortalezcan aquellos aspectos que son valorados al interior de la universidad en los docentes y personal administrativo, y mejorar aquellos que son considerados como área de desarroIlo; de esta manera, se tendrían elementos importantes para trabajar en la marca empleador y contar con los mejores profesionales a nivel de todas las áreas en la institución y a nivel nacional.

\section{JUSTIFICACIÓN}

A nivel nacional, diversas instituciones de educación superior han tenido que cumplir y adaptar un sinnúmero de requerimientos para obtener la certificación y acreditación de las carreras que ofrecen, para de esa forma garantizar su funcionamiento y calidad. La gestión docente se ha visto directamente impacta- da a causa de estos requerimientos por lo que es importante determinar cómo han incidido en la percepción de justicia y compromiso organizacional.

El compromiso organizacional cobra vigencia en el área del talento humano, por el impacto que este tiene en el logro de los objetivos, así como al em- 
poderamiento y desempeño en los colaboradores; por esto es necesario realizar un análisis de los tipos de compromiso. Los factores de justicia y compromiso organizacional se analizarán en una muestra representativa de docentes de varias unidades académicas de la PUCE. Otro factor importante para el análisis es la relación contractual y el tipo de dedicación de los docentes de la PUCE y cómo esto afecta en las percepciones de justicia y compromiso organizacional.

La investigación radica en conocer la percepción de justicia organizacional de los docentes de la PUCE, matriz Quito, de varias facultades; las encuestas se enfocan en evaluar el nivel de justicia en sus tres dimensiones: 1. distributiva; 2. procedimental; 3. informacional e interaccional; y 4. determinar cómo estos factores afectan el compromiso afectivo, normativo y de continuidad.

El diagnóstico de la percepción de justicia y el nivel de compromiso organizacional en los docentes de cada una de las facultades, se realizó mediante un cuestionario de compromiso organizacional que mide los tres tipos de compromiso. También, se utilizó un cuestionario de Percepciones de Justicia Organizacional que mide la justicia distributiva, procedimental interpersonal e informativa. Con la información obtenida se definirán los factores que inciden en la percepción de justicia y compromiso organizacional de los docentes. Posterior a este análisis, se presentaron los resultados y la relación entre los factores de justicia y nivel de compromiso organizacional.

El tema es importante para la PUCE cuando se comprenda si los valores, los procedimientos, las distribuciones y las interacciones actúan como factores determinantes positivos o negativos en las relaciones entre las prácticas organizacionales y las cogniciones, actitudes y comportamientos asociados con el trabajo.

Esta investigación responde a la interrogante de la existencia o no, de una correlación directa entre las variables de justicia organizacional y compromiso organizacional en medio de los cambios a los que los docentes de la PUCE, matriz Quito, se han visto sujetos. Además, se espera que este artículo sea un insumo para futuras investigaciones sobre el tema. 


\section{MARCO TEÓRICO}

\section{Justicia organizacional}

La justicia organizacional se relaciona con las percepciones que pueden existir en los colaboradores frente a aspectos de distribución, procedimientos, la manera en que están siendo tratados e informados en la organización. Los colaboradores realizan de manera inconsciente una comparación de los esfuerzos realizados, frente a los esfuerzos de otros colaboradores, lo que genera un análisis que determina si existe equidad o inequidad; con base a esto sus comportamientos influyen en la satisfacción laboral, motivación, desempeño y productividad.

\section{Orígenes de la justicia}

\section{organizacional: Teoría de la equidad}

La teoría de la Equidad plantea que las personas establecen una ecuación entre los resultados percibidos de su trabajo y su percepción de sus aportes a este trabajo; el resultado de dicha ecuación lo comparan con las ecuaciones de los demás. En el artículo "Justicia Organizacional: Entendiendo la Equidad en las organizaciones", Adams postula que si los resultados de las ecuaciones son iguales, los sujetos se sentirán satisfechos (Mladinic \& Isla, 2002, pág. 172); por el contrario, si el resultado del indivi- duo es diferente al de los otros, esa situación representaría "inequidad".

Con el desarrollo industrial surgen ciertas teorías que se enfocan al estudio de las percepciones y necesidades humanas reflejadas en el ámbito laboral y la relación que existe entre el aporte del colaborador y lo que recibe de la empresa. Si esta relación es percibida como equitativa favorecerá la relación entre la empresa y los colaboradores; caso contrario, se generará un sentimiento de inequidad e injusticia que involucra el compromiso hacia la organización. Por otro lado, una percepción justa de parte de los colaboradores ayuda a una mayor interacción entre ellos.

\section{Justicia distributiva}

Goleman se refiere a la justicia distributiva como la percepción que tiene un individuo sobre lo justo o injusto en relación a los montos y recompensas en un ambiente social y lo relaciona con resultados deseables como satisfacción laboral, compromiso, ciudadanía organizacional, rotación y desempeño (Luthans \& Luthans, 2008).

La percepción de justicia distributiva al ser individual se torna subjetiva, y para contrarrestarla las organizaciones crean mecanismos 
que canalicen objetivamente las percepciones, mediante procedimientos, políticas, indicadores; así, las recompensas, reconocimientos y beneficios que recibe el colaborador se asocian al cumplimiento de metas y objetivos. La distribución de recompensas se basa en el esfuerzo y desempeño del colaborador hacia el logro de resultados.

\section{Justicia procedimental}

La justicia procedimental se relaciona con los métodos usados para tomar decisiones y establecer recompensas en función a los resultados organizacionales, ya que están ligados a la implementación exitosa de cambios. Según varios análisis, la justicia procedimental era un mejor factor de predicción del desempeño laboral que la justicia distributiva (Luthans \& Luthans, 2008).

Un procedimiento es un conjunto de actividades que se realiza de la misma forma, bajo iguales circunstancias para lograr siempre el mismo resultado. Los procedimientos en una organización contemplan ciertas características en su diseño y en su aplicación de tal forma que estos sean beneficiosos tanto para la organización como para el colaborador, y que su aplicación logre el efecto de equidad en la percepción de justicia en los colaboradores.

\section{Justicia interpersonal e informativa}

Colquitt realizó un estudio sobre justicia; los resultados mostraron que la justicia de interacción tiene dos elementos: justicia interpersonal y justicia de información.

La justicia interpersonal es el grado de respeto, amabilidad y dignidad con el que se trata a las personas o colaboradores.

La justicia de información se relaciona con las explicaciones que se proporcionan a las personas sobre los procedimientos y los resultados de una organización.

La justicia procedimental se relaciona con la justicia informacional o interaccional, la diferencia se da por la manera en que son diseñados los procedimientos formales e informales, la forma y la oportunidad en que es transmitida la información en la organización. Para fortalecer la percepción de este tipo de justicia, es necesario la creación de un procedimiento de comunicación interna que tenga varios canales de comunicación que permita conocer a la organización de manera global.

\section{Compromiso organizacional}

El concepto de compromiso laboral incluye la aceptación de las metas de la organización, la disposición a realizar esfuerzos en pro de esta y la ex- 
presión del deseo de mantenerse como miembro activo de la misma, dentro de una práctica constante.

Según Meyer y Allen, en su artículo "El compromiso laboral: discursos en la organización" (Peralta Gómez, Santofimio, \& Segura, 2007) (1991), creen que el compromiso laboral está constituido por tres componentes separables, y cada uno de ellos refleja un estado psicológico único. El compromiso afectivo es la asociación emocional de un empleado con la organización, de manera que este continúa en el empleo porque así lo desea.

El compromiso de continuidad es la conciencia de los costos asociados a dejar la organización, de manera que los empleados permanecen porque consideran que necesitan el empleo. Finalmente, el compromiso normativo es el sentimiento de obligación, debido a lo cual los empleados sienten que deben permanecer en la organización. El concepto de compromiso laboral cobra importancia en las organizaciones debido al interés de retener a los trabajadores en la organización y lo asocian como predictor del desempeño, bajo ausentismo y altos resultados financieros.

El compromiso laboral es una consecuencia de la relación existente entre en la organización y el colaborador; relación que se refleja en la actitud de confianza que tiene el colaborador hacia los valores de la organización, en el esfuerzo que realiza en favor del logro de metas organizacionales y el sentimiento de pertenencia hacia esta que incide en su permanencia en la institución. Al ser las actitudes y emociones positivas, que están asociadas con el compromiso, estas se manifiestan en el incremento del nivel de satisfacción, entusiasmo, alto desempeño, productividad, logro de resultados y bajo nivel de rotación.

\section{Compromiso afectivo}

El compromiso afectivo (deseo) es el lazo emocional que las personas crean con la organización al percibir la satisfacción de sus necesidades y expectativas; por ende, disfrutan de su permanencia en la organización. (Chiang, Nuñez , Martin , \& Salazar, 2010, pág. 91). El bienestar del colaborador no se restringe únicamente al bienestar físico, sino que está asociado a un bienestar integral en donde el bienestar psicológico juega un papel fundamental al momento de generar e incrementar compromiso.

El compromiso afectivo no se relaciona solo con el puesto de trabajo sino con otros aspectos de su entorno laboral como su equipo, jefes, compañeros, entre otros que, si son positivos, pueden generar menor rotación y ausentismo laboral y un mayor nivel de compromiso. 


\section{Compromiso de continuidad}

En el compromiso de continuidad (necesidad) es muy posible que la persona evalué las inversiones en tiempo y esfuerzo que perdería en caso de dejar la organización; es decir, los costos en los que incurriría al retirarse o las pocas posibilidades de encontrar otro empleo. Las personas con un compromiso de continuidad necesitan permanecer en la empresa (Chiang, Nuñez, Martin, \& Salazar, 2010) (p. 91).

\section{Compromiso normativo}

Al compromiso normativo (de- ber) se relaciona con sentimientos de retribución del empleado hacia la institución y se asocia directamente con el compromiso y la permanencia; este sentimiento se da cuando los colaboradores reciben ciertos beneficios y son valorados como importantes; sin embargo, en esta condición intervienen otros factores como la motivación y satisfacción.

Tanto el bienestar físico y emocional están relacionados con el grado de satisfacción y compromiso del colaborador; es decir, a mayor nivel de bienestar físico y emocional, mayor nivel de compromiso.

\section{MÉTODO}

La investigación es de tipo correlacional que mide las variables justicia y compromiso organizacional mediante un procedimiento de muestreo.

Para obtener la información, se utilizaron herramientas de medición para cada una de las variables; estas se aplicaron a los docentes de la PUCE realizando un trabajo de campo.

\section{Elección de la herramienta}

Para el levantamiento de la información se utilizaron dos cuestionarios: 1. El cuestionario de percepciones de justicia organizacional de Colquitt, adaptado al es- pañol, de Ruíz y García (2013) y 2. El cuestionario de escala modificada de compromiso de Meyer, Allen y Smith (1993).

\section{Cuestionarios de justicia organizacional y compromiso}

Percepciones de justicia organizacional: se aplicó el cuestionario de percepciones de justicia organizacional de Colquitt, adaptado al español, de Ruíz y García (2013). El cuestionario tiene cuatro escalas: justicia distributiva, 4 ítems, alfa = 0.90; procedimental, 7 ítems, alfa= 0.71; interpersonal, 4 ítems, alfa $=0.75$; e informativa, 5 ítems, alfa $=0.84$. 
Los participantes respondieron en una escala de tipo Likert de cinco anclajes, con un total de 20 ítems.

\section{Cuestionario de compromiso organizacional}

Se usará la escala modificada de compromiso de Meyer, Allen y Smith (1993) que mide los tres tipos de compromiso: afectivo, normativo y de continuidad. Cada subescala tiene 6 ítems con un total de 18 preguntas y un formato de calificación. Una ventaja del cuestionario es que se obtienen tres puntuaciones que corresponden a cada uno de los tipos de compromisos planteados por Meyer y Allen (1997) y la posibilidad de obtener un perfil de compromiso. Los índices alfas de las escalas han sido variadas en diferentes estudios, donde la escala de compromiso de con- tinuidad suele reportar los alfas más bajos (Arias 2001; Ko, Price y Mueller, 1997; Littlewood, 2006). Los alfas promedios reportados para cada escala son: 0.85 , 0.83 y 0,77 respectivamente.

Para la validación de los cuestionarios, se realizó una prueba piloto con docentes de la facultad de Psicología, con el objetivo de verificar posibles inconsistencias en los instrumentos. El resultado de la prueba piloto fue favorable; existieron recomendaciones para modificar cierta terminología. Efectuados los cambios, se procedió a la aplicación de los mismos en la muestra.

En la recolección de datos, se identificaron datos sociodemográficos como género, edad, facultad a la que pertenece, tipo de dedicación, tipo de categoría y antigüedad.

\section{CARACTERIZACIÓN DE LA MUESTRA}

La muestra de esta investigación nidos, ésta puede ser considerada reprefue de tipo aleatorio. Este procedimiento muestral selecciona un subgrupo de la población y a partir de los datos obte-

sentativa de toda la población.

Para obtener la muestra se aplicó la siguiente fórmula (Espejo, y otros, 2009):

$$
n=\frac{N \sigma^{2} z^{2}}{(N-1) E^{2}+\sigma^{2} z^{2}}
$$


La equivalencia de los elementos de la fórmula muestral son los siguientes:

Tabla 3.1 Equivalencia muestral

\begin{tabular}{|c|c|}
\hline$n$ & Tamaño de la muestra \\
\hline$N$ & Población \\
\hline$\sigma$ & Varianza de la población \\
\hline$Z$ & Nivel de confiabilidad \\
\hline$E$ & Error muestral \\
\hline IC & Índice de confiabilidad \\
\hline
\end{tabular}

Realizando la fórmula correspon- tados: diente se obtienen los siguientes resul-

\begin{tabular}{|l|l|}
\hline$N$ & 1200 docentes \\
\hline$n$ & 291 docentes \\
\hline
\end{tabular}

La población de docentes de la PUCE, matriz Quito, a noviembre del 2015, fue de aproximadamente 1200 docentes, con diferentes tipos de dedicación, categoría y de varias facultades, según datos proporcionados por la APPUCE.

Con la aplicación de la fórmula, se establece la realización de 291 encuestas, de las cuales se aplicaron efectivamente 278. El nivel de confianza que representan las 291 encuestas es del $95 \%$; al contar con 278 encuestas, el nivel de confianza disminuye al $94.26 \%$, sin que esto invalide la confiabilidad de los datos, ya que el índice sigue estando dentro de los parámetros del coeficiente de seguridad, lo que implica que es una muestra válida y representativa.

En su libro Estadística aplicada a las Ciencias Sociales tamaño necesario de la muestra: ¿Cuántos sujetos necesitamos?, Pedro Morales Vallejo, de la Facultad de Humanidades de la Universidad Pontificia Comillas de Madrid, establece que se puede extrapolar los resultados obtenidos cuando los datos cumplen con las siguientes variables: nivel de confianza, varianza y margen de error. (Morales VaIlejo, 2011, págs. 4-5). Esta condición se cumple en la investigación realizada de acuerdo a los siguientes resultados: 


\begin{tabular}{|c|l|}
\hline$n$ & 278 \\
\hline$N$ & 1200 \\
\hline$\sigma^{2}$ & 0.25 \\
\hline$Z^{2}$ & $1.9^{2}$ \\
\hline$(N-1)$ & Coeficiente de correlación \\
\hline$E^{2}$ & 0.0025 \\
\hline $\mathrm{IC}$ & $94.26 \%$ \\
\hline
\end{tabular}

Procedimiento para la recolección de datos

En varias facultades de la PUCE, matriz Quito, se informó sobre el objetivo de la investigación, se solicitó la participación y se realizó una explicación de cómo completar los cuestionarios.
Se presentó la autorización de la DGA y además se entregó el consentimiento informado, donde se evidencia la participación libre y voluntaria del docente. El proceso de recolección de datos tomó un tiempo aproximado de dos meses. 
Tabla 3.3 Número de encuestas realizadas por facultad.

\begin{tabular}{|c|c|c|}
\hline FACULTAD & NÚMERO DE ENCUESTAS & $\%$ \\
\hline Ciencias administrativas y contables & 42 & $15,11 \%$ \\
\hline Comunicación, lingüística y literatura & 39 & $14,03 \%$ \\
\hline Psicología & 39 & $14,03 \%$ \\
\hline Medicina & 32 & $11,51 \%$ \\
\hline Enfermería & 24 & $8,63 \%$ \\
\hline Ciencias exactas y naturales & 20 & $7,19 \%$ \\
\hline Ciencias humanas & 18 & $6,47 \%$ \\
\hline Arquitectura, diseño y artes & 14 & $5,04 \%$ \\
\hline Ingeniería & 12 & $4,32 \%$ \\
\hline Jurisprudencia & 12 & $4,32 \%$ \\
\hline Economía & 10 & $3,60 \%$ \\
\hline Ciencias de la educación & 8 & $2,88 \%$ \\
\hline Ciencias filosófico-teológicas & 5 & $1,80 \%$ \\
\hline Escuela de bioanálisis & 2 & $0,72 \%$ \\
\hline En blanco & 1 & $0,36 \%$ \\
\hline TOTAL & 278 & $100 \%$ \\
\hline
\end{tabular}

\section{RESULTADOS}

El análisis de resultados se realizó mediante estadística descriptiva y correlacional.

\section{Coeficientes de Cronbach}

Los coeficientes de Cronbach de los ítems del cuestionario de compromiso y justicia organizacional son: 
Tabla 4.1 Coeficientes de Cronbach de compromiso organizacional.

\begin{tabular}{ccc}
\hline Escala & \# Ítems & Alfa \\
\hline Compromiso global & 18 & 0,89 \\
Compromiso afectivo & 6 & 0,74 \\
Compromiso normativo & 6 & 0,91 \\
Compromiso de continuidad & 6 & 0,77 \\
\hline
\end{tabular}

Tabla 4.2 Coeficientes de Cronbach de justicia organizacional

\begin{tabular}{ccc}
\hline Escala & \# Ítems & Alfa \\
\hline Justicia global & 20 & 0,95 \\
\hline Justicia procedimental & 7 & 0,93 \\
\hline Justicia distributiva & 4 & 0,91 \\
\hline Justicia interpersonal & 4 & 0,96 \\
\hline Justicia informativa & 5 & 0,95 \\
\hline
\end{tabular}

De acuerdo a los coeficientes obtenidos en todas las escalas y factores, se determina que existe una fiabilidad interna, lo que garantiza la validez de los instrumentos utilizados.

\section{Contraste}

La hipótesis planteada: "A mayor percepción de justicia organizacional, mayor nivel de compromiso organizacional", determina que es una hipótesis direccional, ya que se especifica una relación positiva entre las variables XY; para lo cual se realizó un contraste unilateral o de una cola en sistema SPSS.

Según los análisis, se contrasta favorablemente la hipótesis ya que existe una correlación directa positiva, pero con una magnitud baja, entre la variable de justicia organizacional en sus cuatro dimensiones (procedimental, distributiva, interpersonal e informativa), con la variable compromiso organizacional en dos de sus dimensiones (afectivo y de continuidad). 


\section{Análisis de correlación}

El concepto de relación o correlación entre dos variables se refiere al grado de parecido o variación conjunta existente entre las mismas (Informática).

Correlación unilateral: Probabilidad de obtener un resultado tan extremo como el observado y en la misma dirección, cuando la hipótesis nula es cierta. Contrasta la hipótesis nula en la que se especifica con antelación la dirección del efecto.

El sistema SPSS por defecto mar- ca las correlaciones significativas con un asterisco y los coeficientes al nivel 0,05 y, con dos asteriscos, las correlaciones significantes al nivel 0,01 .

Se dice que la correlación entre dos variables $X$ e $Y$ es perfecta positiva cuando exactamente en la medida que aumenta una de ellas aumenta la otra. Esto sucede cuando la relación entre ambas variables es funcionalmente exacta.

Una relación es perfecta negativa cuando exactamente en la medida que aumenta una variable disminuye la otra.

\section{Tabla 4.3 Correlación global}

\begin{tabular}{|c|c|c|c|}
\hline \multicolumn{2}{|c|}{ Correlación global } & Compromiso & Justicia \\
\hline \multirow{3}{*}{ Compromiso } & Correlación de Pearson & 1 & $0,314^{* *}$ \\
\hline & Sig. (unilateral) & & 0,000 \\
\hline & $\mathrm{N}$ & 278 & 278 \\
\hline \multirow{3}{*}{ Justicia } & Correlación de Pearson & $0,314^{* *}$ & 1 \\
\hline & Sig. (unilateral) & 0,000 & \\
\hline & $\mathrm{N}$ & 278 & 278 \\
\hline
\end{tabular}

** $p<0.001$. La correlación es significativa al nivel 0,01 (unilateral).

La correlación global es de 0,314, las dos variables no tienen exactamente lo que determina que es significativa po- los mismos valores, sin embargo, si existe sitiva al nivel 1, sin embargo, la intensi- una correlación en sentido directo. dad se la considera media baja, es decir 


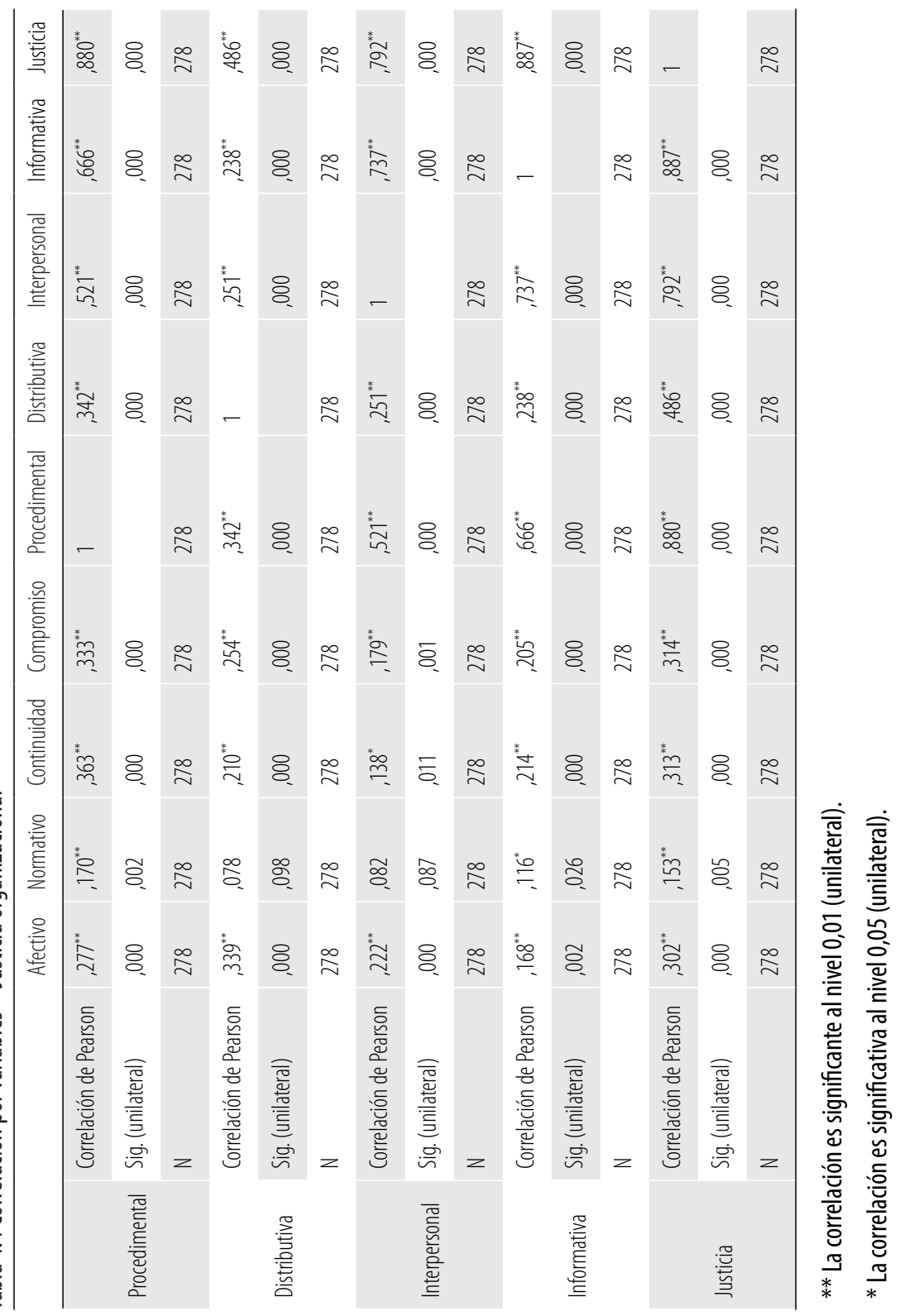




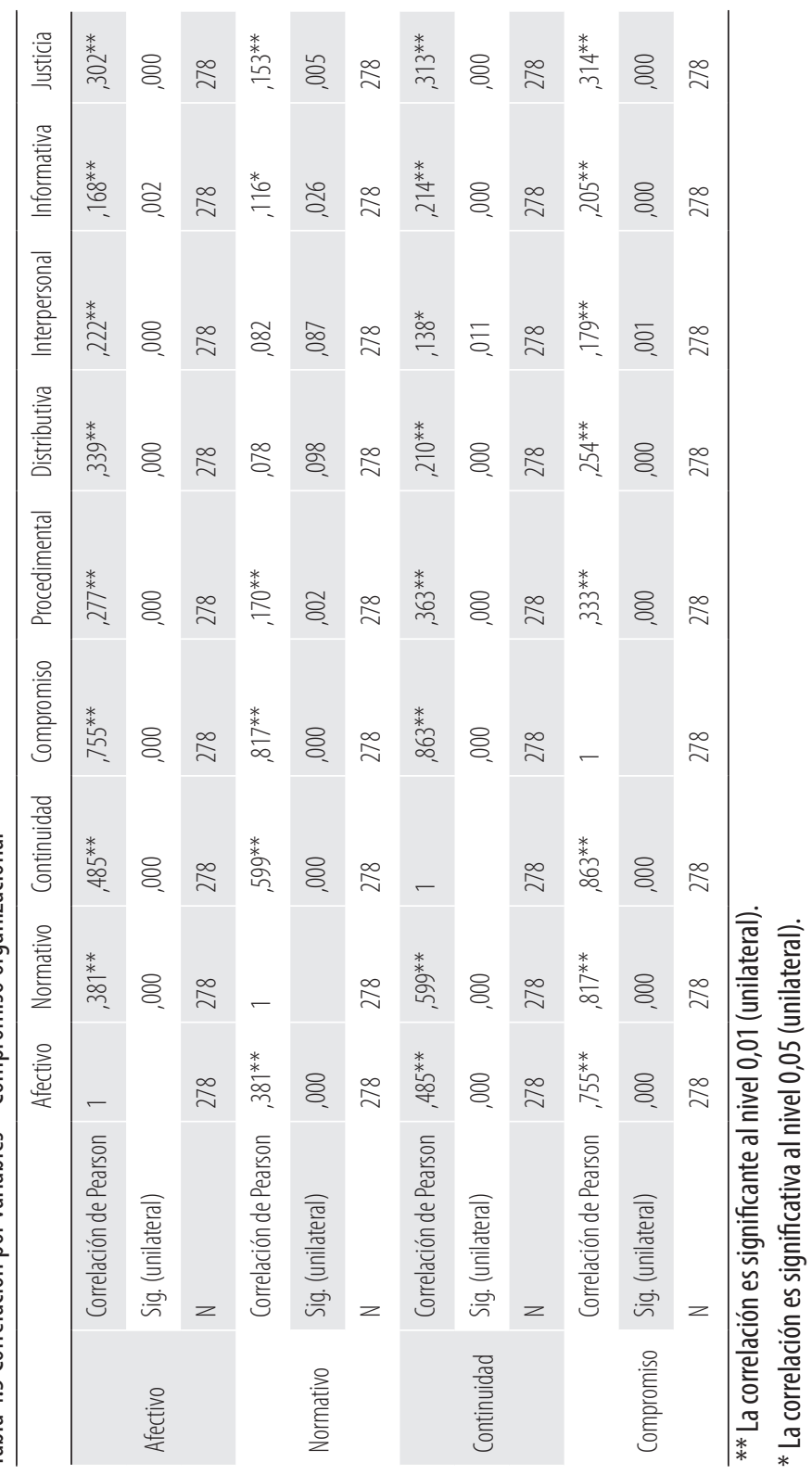


Los datos de la tabla 10 indican que las variables justicia y compromiso organizacional se asocian en alguna medida; sin embargo, se evidencia que existe una magnitud pequeña.

En las variables se determinó que existe una correlación significante más alta, pero con una magnitud bastante baja entre: Justicia procedimental y Justicia distributiva.

Justicia procedimental con compromiso de continuidad, en relación a la implicación de estas variables se puede señalar que a medida que el docente percibe que las normas y políticas implementadas en la PUCE se fundamentan en valores éticos y morales; que las reglas y procedimientos para la toma de decisiones son aplicados consistentemente, y considerando los puntos de vista de los docentes, con información correcta, evitando que las reglas y procedimientos sean tendenciosos o arbitrarios, se genera una percepción de justicia positiva, contribuyendo a que exista un mayor nivel de compromiso de continuidad por parte de los docentes.

Justicia distributiva con compromiso afectivo, en este caso cuando los docentes perciben que los resultados alcanzados reflejan el esfuerzo realizado desde su puesto de trabajo y la verdadera importancia y contribución que esto tiene hacia la PUCE, se logra un mayor nivel de compromiso afectivo.

\section{Regresiones}

Se realizó un análisis de regresión con las variables de compromiso organizacional, considerando como dependientes para determinar si los niveles de compromiso se pueden predecir a partir de las percepciones de justicia organizacional y viceversa; es decir, si las percepciones de justicia se pueden pronosticar a partir de los tipos de compromiso. En la ejecución de los análisis no se encontraron modelos predictivos significativos.

\section{Frecuencia en las variables compromiso organizacional y justicia organizacional}

Se estandarizaron los puntajes de cada escala de la muestra, para poder comparar con cada una de las personas y ver qué tipo de compromiso y justicia predomina y se desarrolla mayormente en los docentes encuestados. 
Figura 4.1 Frecuencia de justicia organizacional

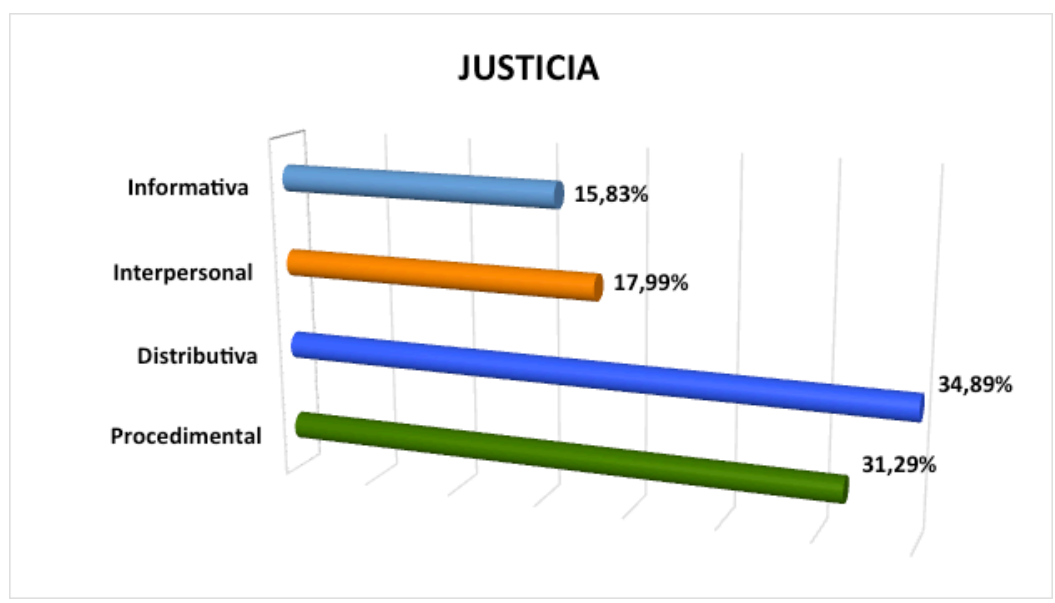

En el caso de justicia organiza- toridades de la PUCE; sus actitudes son cional existe mayor frecuencia en la di- positivas frente a las disposiciones y dismensión de justicia distributiva; es decir, minuyen las reacciones negativas frente existe un grado alto de aceptación en posibles percepciones de injusticia. las decisiones de los jefes directos y au-

Figura 4.2 Frecuencia de compromiso organizacional

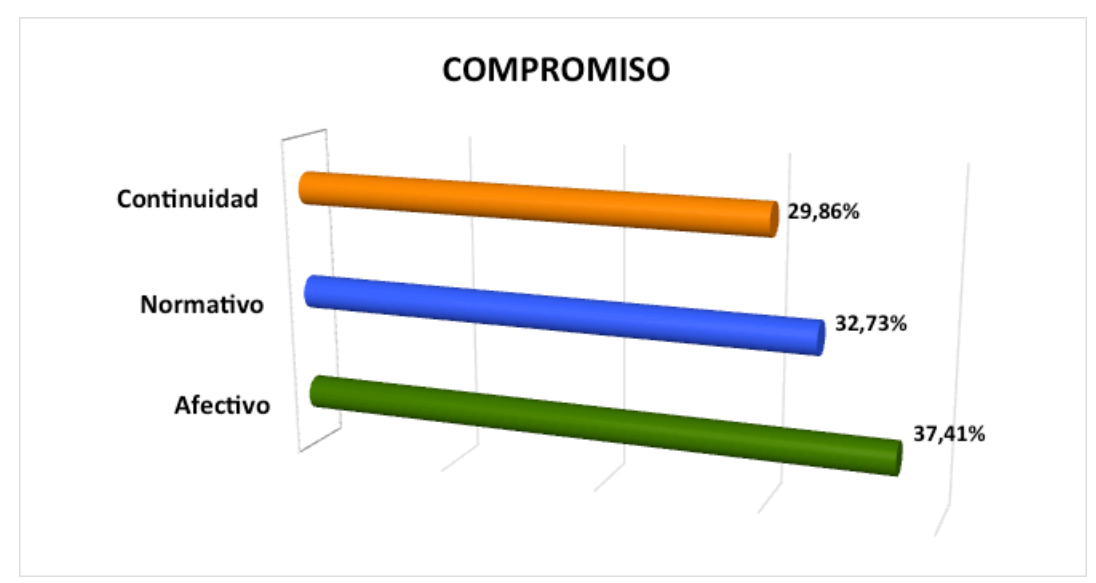


En la variable de compromiso organizacional existe una mayor frecuencia en la dimensión de compromiso afectivo, determinando que los docentes mantienen un apego emocional hacia la universidad, ya que sus necesidades de seguridad en gran parte han sido cubiertas, lo cual ocasiona que permanezcan en la institución con sentido de pertenencia y agrado.

\section{Análisis de datos demográficos}

Figura 4.3 Participación de docentes según su género

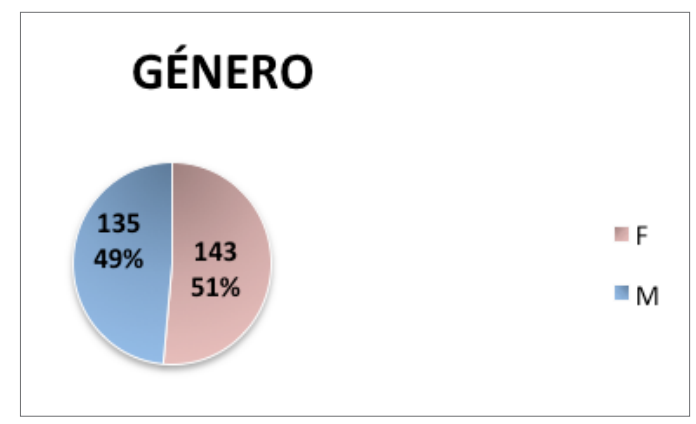

Figura 4.4 Docentes por edades

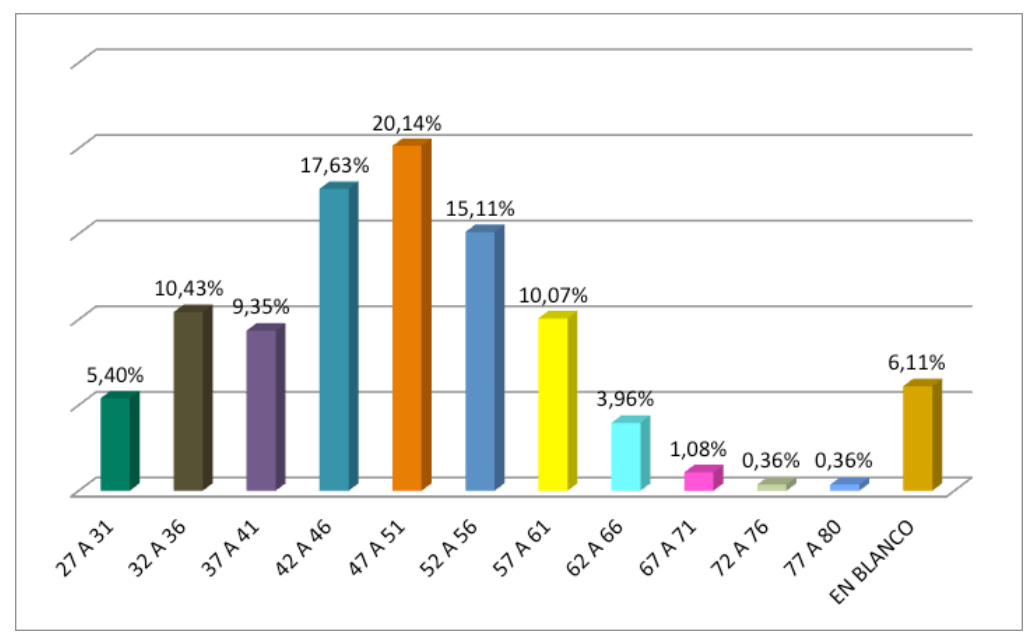


Figura 4.5 Tipo de dedicación de los docentes

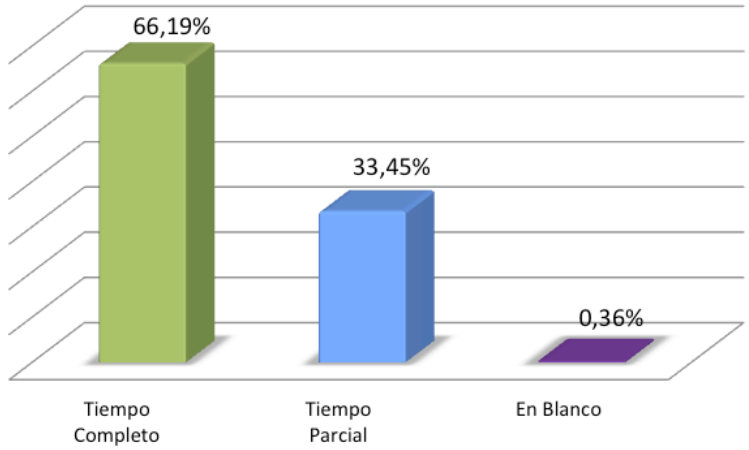

Figura 4.6 Docentes por antigüedad.

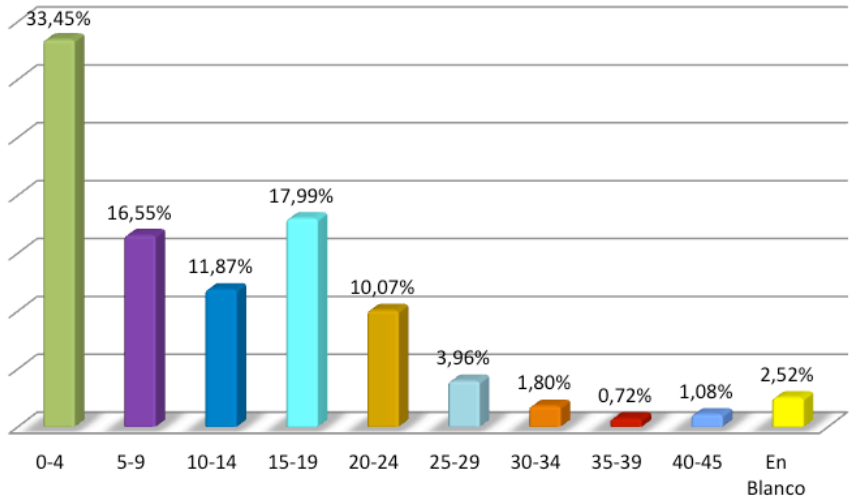


Tabla 4.8 Antigüedad por tipo de categoría-agregado

\begin{tabular}{ccc}
\hline RANGOS ANTIGÜEDAD & AGREGADO & $\%$ \\
\hline (en blanco) & 1 & $0,36 \%$ \\
\hline $0-4$ & 20 & $7,19 \%$ \\
\hline $5-9$ & 8 & $2,88 \%$ \\
\hline $10-14$ & 13 & $4,68 \%$ \\
\hline $15-19$ & 24 & $8,63 \%$ \\
\hline $20-24$ & 18 & $6,47 \%$ \\
\hline $25-29$ & 2 & $0,72 \%$ \\
$30-34$ & 2 & $0,72 \%$ \\
$40-45$ & 1 & $0,36 \%$ \\
\hline Total general & 89 & $32,01 \%$ \\
\hline
\end{tabular}

Tabla 4.9Antigüedad por tipo de categoría-auxiliar

\begin{tabular}{ccc}
\hline RANGO DE ANTIGÜEDAD & AUXILIAR & $\%$ \\
\hline (en blanco) & 2 & $0,72 \%$ \\
\hline $0-4$ & 60 & $21,58 \%$ \\
\hline $5-9$ & 28 & $10,07 \%$ \\
\hline $10-14$ & 14 & $5,04 \%$ \\
$15-19$ & 2 & $0,72 \%$ \\
$20-24$ & 1 & $0,36 \%$ \\
\hline $25-29$ & 2 & $0,72 \%$ \\
\hline Total general & 109 & $39,21 \%$ \\
\hline
\end{tabular}

Tabla 4.10 Antigüedad por tipo de categoría-ocasional

\begin{tabular}{ccc}
\hline RANGO DE ANTIGÜEDAD & OCASIONAL & $\%$ \\
\hline $0-4$ & 3 & $1,08 \%$ \\
$5-9$ & 2 & $0,72 \%$ \\
\hline Total general & 5 & $1,80 \%$ \\
\hline
\end{tabular}


Tabla 4.11 Antigüedad por tipo de categoría - principal

\begin{tabular}{ccc}
\hline RANGO DE ANTIGÜEDAD & PRINCIPAL & $\%$ \\
\hline (en blanco) & 3 & $1,08 \%$ \\
$0-4$ & 2 & $0,72 \%$ \\
\hline $5-9$ & 1 & $0,36 \%$ \\
\hline $10-14$ & 4 & $1,44 \%$ \\
\hline $15-19$ & 23 & $8,27 \%$ \\
\hline $20-24$ & 9 & $3,24 \%$ \\
\hline $25-29$ & 7 & $2,52 \%$ \\
\hline $30-34$ & 3 & $1,08 \%$ \\
$35-39$ & 2 & $0,72 \%$ \\
\hline $40-45$ & 2 & $0,72 \%$ \\
\hline Total general & 56 & $20,14 \%$ \\
\hline
\end{tabular}

\section{CONCLUSIONES}

Se confirma la hipótesis planteada: "A mayor percepción de justicia organizacional, mayor nivel de compromiso organizacional", ya que existe una correlación directa positiva con una magnitud baja entre las dos variables: justicia y compromiso organizacional.

El tipo de compromiso que predomina en los docentes de la PUCE es el compromiso afectivo, lo que le otorga a la universidad docentes con mayor estabilidad laboral, aceptación de metas, menor ausentismo, baja rotación de personal y mayor satisfacción laboral.
El tipo de percepción que predomina en los docentes de la PUCE es la de justicia distributiva, lo cual favorece el aumento del grado de aceptación en las decisiones tomadas por sus jefes directos y autoridades de la PUCE; por tanto, mantienen actitudes positivas frente a las disposiciones y disminuyen reacciones negativas ante posibles percepciones de injusticia.

Existe una correlación positiva y significante a nivel 0,01 entre la percepción de justicia procedimental y el compromiso organizacional en sus tres 
dimensiones: afectivo, normativo y de continuidad, lo cual implica que los docentes perciban que las normas y procedimientos aplicados en la PUCE se fundamentan en valores éticos y morales. La toma de decisiones se basa en reglas y procedimientos establecidos que son aplicados consistentemente considerando los puntos de vista de los docentes. Las reglas y procedimientos son objetivos, con información correcta, y se basan en la razón, de manera lógica y con justicia.

Existe una correlación positiva y significante a nivel 0,01 entre la percepción de justicia distributiva y el compromiso organizacional en dos de sus dimensiones: afectivo y de continuidad; lo que significa que los docentes perciben que los resultados alcanzados reflejan el esfuerzo realizado desde su puesto de trabajo y la importancia de su contribución hacia la PUCE.

Existe una correlación positiva y significante a nivel 0,01, entre la percepción de justicia interpersonal con compromiso afectivo (deseo) y una correlación positiva significativa a nivel 0,05 con compromiso de continuidad (necesidad), lo cual evidencia que los docentes perciben que son tratados con cortesía, dignidad, respeto y que no se generan chistes o comentarios inapropiados por parte de su jefe directo.

Existe una correlación positiva y significante a nivel 0,01 entre las per- cepciones de justicia informativa y compromiso organizacional en dos de sus dimensiones: afectivo y de continuidad. Por otro lado, existe una correlación significativa a nivel 0,05 entre la percepción de justicia informativa y compromiso normativo, lo cual determina que los docentes sienten que sus necesidades son consideradas; la comunicación con su jefe directo es sincera, oportuna, con claridad y detalle frente a las decisiones.

El resultado de estas percepciones positivas de justicia organizacional en sus cuatro dimensiones: procedimental, distributiva, interpersonal e informativa, ha generado en los docentes compromiso organizacional en dos de sus dimensiones: el afectivo (deseo) y el de continuidad (necesidad).

En el compromiso afectivo se demuestra que los docentes tienen un apego emocional hacia la universidad, sentimiento que se ha logrado como consecuencia de tener cubiertas sus necesidades, especialmente las psicológicas, lo que les permite disfrutar de su permanencia en la institución y sentirse orgullosos de permanecer en ella.

Así, también, se concluye que existe un compromiso positivo de continuidad (necesidad) debido a la inversión de tiempo, dinero y esfuerzo por parte de los docentes hacia la universidad. Consideran, además, los costos financieros, físicos y psicológicos que implicaría 
renunciar a la PUCE; esto genera, por tanto, mayor apego a la universidad.

La percepción positiva de justicia procedimental respecto al compromiso normativo (deber), logra que los docentes cumplan su trabajo con lealtad, obligación moral como consecuencia de un sentimiento de reciprocidad frente a una oportunidad o recompensa entregada por la universidad a los docentes y que, al ser valorada por ellos, desarrolla un fuerte sentimiento de permanencia en la institución.

Analizadas varias investigaciones sobre el tema de compromiso y justicia organizacional, se concluye que los resultados obtenidos en dichas investigaciones son similares a los resultados de la presente; esta afirmación se basa en las conclusiones, donde se señalan que los colaboradores al percibir que son tratados de manera justa, equitativa y que cuentan con condiciones de trabajo favorables, genera en el personal compromiso de continuidad en mayor o menor nivel; así mismo, se logra fortalecer el compromiso afectivo con el trabajo y la institución, lo cual, se lo evidencia por el comportamiento de ciudadanía organizacional que demuestran los colaboradores. 


\section{BIBLIOGRÁFIA}

Alcover de la Hera, C. (2012). Psicología del Trabajo. Obtenido de http:// puceftp.puce.edu.ec:2057/lib/ pucesp/13314\&ppg=12

Arias Galicia, F., Valera Loza, D., Loli Pineda, A., \& Quintana Otiniano, M. (s.f.). El Clima Organizacional y el Compromiso Personal hacia la Organización, la intención de Permanencia y el Esfeurzo: Evidencias provenientes de Perú. Revista Liberabit, 11 - 24.

Arias, G. (2001). El compromiso personal hacia la organización y la intención de permanencia: algunos factores para su incremento. Contaduría y Administración, 5-12.

Baron, R. A., \& Byrne, D. (2005). Psicología Social (Décima ed.). Madrid: España.

Betanzos Díaz, N., \& Paz Rodríguez, F. (2007). Análisis psicométrico del compromiso organizacional como variable actitudinal. Anales de Psicología, 1 - 9.

Betanzos Díaz, N., Andrade Palos, P., \& Paz Rodríguez, F. (2006). Compromiso organizacional en una muestra de trabajadores mexicanos. Revista de Psicología del Trabajo y de las Organizaciones, 1 - 20.

Blanco, M., \& Castro Ch, P. (Agosto de 2011). Análisis comparativo del compromiso organizacional. Obtenido de http://www.ucb.edu. bo/publicaciones/ajayu/v9n2/ v9n2a2.pdf

Böhrt, R., Romero, C., \& Díaz Bretones, F. (2014). El compromiso organizacional entre obreros: un estudio en cuatro organizaciones bolivianas. Scielo.

Celis Muñoz, A. A., \& García Valerio, A. (s.f.). Trabajo presentado para el Premio Nacional de Investigación del COLPARMEX 2008 en el área de Administración. UMSA, 1 - 44.

Chiang, M., Nuñez , A., Martin , M., \& Salazar, M. (2010). Compromiso del Trabajador hacia su Organización y la Relación con el Clima Organizacional: Un Análisis de Género y Edad. Panorama Socioeconómico, $92-103$.

Chiavenato, I. (2009). Comportamiento Organizacional. La Dinámica del Éxito en las Organizaciones. México: Mc Graw Hill.

Córdova Pirela, C. E. (s.f.). biblioteca2.ucab. edu.ve. Obtenido de http://biblioteca2.ucab.edu.ve/anexos/biblioteca/marc/texto/AAQ4704.PDF

Dávila de León, C., \& Jiménez García, G. (2014). Sentido de pertenencia y compromiso organizacional: predicción del bienestar. Revista de 
Psicología (PUCP), 1 - 302.

Delgado Abella, L. E., \& Vanegas García, M. (2013). Psicología Organizacional: Perspectivas y Avances. En L. E. Delgado Abella, \& M. Vanegas García, Psicología Organizacional: Perspectivas y Avances. Bogotá: Ecoediciones.

Espejo, I., Fernández, F., López , M., Muñoz, M., Rodríguez, A., Sánchez, A., y otros. (2009). Estadística Descriptiva y Probabilidad. Cádiz: Universidad de Cádiz.

Fernández Palma, M. (2009). La evaluación de desempeño, la percepción de justicia y las reacciones de los empleados. Contabilidad y Negocios, 45.

Fincowsk, F., B., E., \& Krieger de, M. J. (2012). Comportamiento Organizacional. Obtenido de http://site. ebrary.com/lib/pucesp/detail.action?doclD=10957677

Fonseca Herrera, J. M., \& Chacón Gutiérrez, L. (s.f.). Elaboración y validación de un instrumento para medir el compromiso organizacional. XII Encuentro Participación de la Mujer en la Ciencia, 1 - 4.

Franklin , E., \& Krieger, M. (2011). Comportamiento Organizacional Enfoque para América Latina. México: Prentice Hall.

Garrido, A., \& Alvaro, J. L. (2007). Psicología social: perspectivas psicológicas y sociológicas. Madrid: McGraw-Hill. Guizar, R. (2013). Desarrollo Organizacional: Principios y Aplicaciones. México: Mc Graw Hill.

Heinz, K. (2001). Diccionario Enciclopédico de Sociología. Barcelona: Herder.

Informática, E. S. (s.f.). Correlaciones con SPSS. Prácticas de Estadística, 1.

Landy, F. J., \& Conte, J. M. (2006). Psicología Industrial. México: McGrawHill Interamericana.

Loli Pineda, A. (2006). Compromiso organizacional de los trabajadores de una universidad pública de Lima y su relación con algunas variables demográficas. Revista IIPSI, $37-67$.

Luthans, \& Luthans, F. (2008). Comportamiento Organizacional. México: McGraw-Hill.

Madero Gómez, S. M., \& Flores Zambada, R. (2009). Predictores no Financieros del Compromiso de Continuidad: Un Estudio Cuasi - Experimental. La Investigación Administrativa, 7 - 22.

Maldonado Radillo, S. E., Guillén Jiménez , A. M., \& Carranza Prieto , R. E. (2012). El Compromiso Organizacional del Personal Docente y Administrativo de una Universidad Pública . Social Science Research Network, 135 - 142.

Maldonado Radillo, S. E., Ramírez Barón, M. C., García Rivera, B. R., \& Chairez 
Venegas, A. (2014). Compromiso Organizacional de los profesores de una Universidad Púbica. Tecnológica, 12-18.

Manrique de Lara, P. Z. (2009). La Anomia como Moderador de la Relación entre Percepciones de Justicia Organizativa y el Uso Negligente de Internet en el Trabajo. Revista de Psicología del Trabajo y de las Organizaciones, 1 - 13.

Meyer , J., Allen, N., \& Smith, C. (1993). Commitment to organizations and occupations: Extension and test of a three-component conceptualization. Journal of Applied Psychology.

Mladinic, A., \& Isla, P. (2002). Justicia Organizacional: Entendiendo la Equidad en las Organizaciones. PSYKHE, 171 - 179.

Morales Vallejo, P. (2011). Tamaño necesario de la muestra: ¿Cuántos sujetos necesitamos? Estadística aplicada a las Ciencias Sociales, 4 $-5$.

Myers, D. G. (2005). Psicología Social. Bogotá: Mc Graw Hill.

Patlán Pérez, J., Flores Herrera, R., Martínez Torres, E., \& Hernández Hernández, R. (2014). Validez y confiabilidad de la escala de justicia organizacional de Niehoff y Moorman en población mexicana. Scielo.
Peralta Gómez, M., Santofimio, A., \& Segura, V. (2007). El compromiso laboral: Discursos en la Organización. Psicología desde el Caribe, $81-109$.

Pérez Arenas, D. V. (2013). Prácticas de contratación y compromiso organizacional del personal asistencial profesional de las Instituciones Prestadoras de Servicios de Salud privadas niveles III y IV en el municipio de Neiva - Huila en el período 2009 - 2010. Dspace, 1 - 138. PONTIFICIA UNIVERSIDAD CATÓLICA DEL ECUADOR. (2011-2015). PUCE. Obtenido de http://www.puce. edu.ec/portal/content/Universi$\mathrm{dad} / 104$ ?link=oln30.redirect

Ramírez, M. G., \& Ibarra Velásquez, L. A. (2009). http://www.eumed.net/ libros-gratis/2012a/1158/compromiso_organizacional.html. Obtenido de www.eumed.net

Robbins, S. (2004). Comportamiento Organizacional. México: Pearson Education.

Robbins, S. P. (1995). Comportamiento Organizacional. México: Pretice Hall Hispanoamericana, S.A.

Robbins, S., \& Judge, T. (2013). Comportamiento Organizacional. México: Person Educación.

Rodríguez Montalbán, R. L., Salanova Soria, M., \& Martínez Lugo, M. (2014). Justicia organizacional, 
engagement en el trabajo y comportamientos de ciudadanía organizacional: una combinación ganadora. Revista Javeriana, 15 $-28$.

Rosario-Hernáandez, E., \& Millán, L. V. (2011). La relación entre las manifestiaciones de conductas de ciudadanía organizacional con los cinco factores de personalidad, satisfacción laboral, compromiso organizacional, justicia organizacional y contrato psicológico. Revista Puertorriqueña de Psicología.

Ruíz, , M., \& García, M. (2013). Adaptación lingüística y validación del Cuestionario de Justicia Organizacional de Colquitt con una muestra de trabajadores colombianos. En Psicogente, 65-83.

Salvador Ferrer, C. M. (2005). Validez predictiva de los componentes del compromiso del cliente y su rela- ción con la satisfacción. Anales de Psicología, 316 - 321.

Salvador Ferrer, C. M., \& García López, E. (2010). Compromiso e inteligencia emocional en mediadores del Poder Judicial de Oaxaca, México. Diversitas: Perspectivas en Psicología, 1 - 11.

Sarrió Catalá, M., \& Barbera Heredia, E. (1997). Equidad, Justicia y Motivación Humana. Revista de Historia de la Psicología, 355 - 366.

Solano Silva, D. (10 de 2011). Sistemas Administrativos. Obtenido de http:// www.academia.edu/

Uribe, J. (2015). Clima y Ambiente Organizacional: Trabajo, Salud y Factores Psicosociales. Obtenido de http://puceftp.puce.edu.ec:2057/ lib/pucesp/detail.action?do$\mathrm{CID}=11013695$

Vaamonde, J. D. (2013). Justicia Organizacional. Sedici, 171 - 173. 